\title{
Zigbee Based Underground Mines Parameter Monitoring System for Rescue and Protection
}

\author{
Pranoti Anandrao Salankar ${ }^{1}$, Sheeja S. Suresh ${ }^{2}$ \\ ${ }^{I}$ M.TECHStudent (Vlsi Design), Department of Electronics \& Telecommunication Engineering \\ G.H. Raisoni Institute of Engineering and Technology for Women, RTMNU Nagpur University, Nagpur, India \\ ${ }^{2}$ Assistant Professor, Department of Electronics \&Telecommunication Engineering \\ G.H. Raisoni Institute of Engineering and Technology for Women, RTMNU Nagpur University, Nagpur, India
}

\begin{abstract}
This paper based on continuous monitoring underground coal mines parameter such as carbon monoxide, temperature, water level and use wireless Zigbee technology for communication. A microcontroller based system is used for collecting and storing data using respective sensors and making decision accordingly, based on which the mine worker is informed through different alarm tone as well as LED display system. The communication system is reliable based on zigbee, IEEE 802.15.4 standard. This is used for transmission between the hardware circuit fitted in the local site(mines) and the remote monitoring site (computer)through routers. This system is highly beneficial for rescue and protection of miners.
\end{abstract}

Keywords: Rescue System, Router System, Sensors, Underground Miners Safety, Zigbee.

\section{Introduction}

Safety is the most vital part of any type of industry. In the mining industry safety and security is a fundamental aspect of all. To avoid any types of unwanted phenomena, all mining industry follows some basic precaution. Communication is the most vital key factor today, to monitor different parameters such as temperature, increasing water level, and carbon monoxide gas continuously using sensors such as LM35, water level indicator and carbon monoxide gas sensor MQ7 and to take necessary actions accordingly to avoid any types of hazards related to security using buzzer. To enhance safety in underground mines, a reliable communication system must be established between workers, moving in the mine, and a fixed base station. The communication network must not be interrupted at any moment and at any condition. Inside underground mines, the wired communication network system is not so effective. Inside mines due to uncomfortable situation the installation cost as well as maintenance cost is high for wired communication networks. For the successfully wireless data transmission, in this work the zigbeeCC2500 specification is utilized in routers. A cost effective zigbeeCC2500 based wireless mine supervising system with early-warning intelligence on carbon monoxide, temperature, increasing water level in mining area is proposed in.

\section{Objective}

1) Monitoring: To monitor the parameters like carbon monoxide, temperature and water level detection.

2) Communication: Monitored data will transmit toward pe side (receiver unit) through zigbee wireless communication.ZigbeeCC2500 can be used inside mines at routers.

3) Rescue: Whenever the sensor data exceeds the specified threshold (preset) value of temperature, carbon monoxide and water level, the zigbee module at remote monitoring site will transmit alert signal to local site by blowing buzzer continuously and any one of the ' $\mathrm{G}, \mathrm{L}, \mathrm{C}$ ' alphabets will be displayed on LED segment according to the emerging situation ( $\mathrm{G}$ for gas, $\mathrm{L}$ for increasing water level, $\mathrm{C}$ for temperature).If monitoring unit wants to call urgently to any employee, then buzzer will ring twice and the employee number will be displayed on LED display. For example in bank account counter.

4) Protection: Due to this wireless communication system, employees will be alert earlier as compare to present underground system. So rapid action will be taken by rescue team. So the chances of accidents will be reduced due to the underground mines environmental factors.

\section{Underground Mines Wireless Network Design}

1) Zigbee Wireless Technology:The ZigBee protocol is the only international IEEE 802.15.4standard wireless sensor network protocol in existence, catering to the specific needs of low-power, low-cost, low maintenance monitoring and control systems with talks of using it in sensor networks [4]. The name refers to the waggle dance of honey bees after their return to the beehive [6]. The network layer supports three topologies: star, cluster tree and mesh. Direct sequence spread-spectrum at $2.4 \mathrm{GHz}$ (ISM), $915 \mathrm{MHz}$ (the United States) and 868 $\mathrm{MHz}$ (Europe) is applied in industrial, scientific and medical frequency band [4]. 
2) Zigbee module (Trans receiver module):

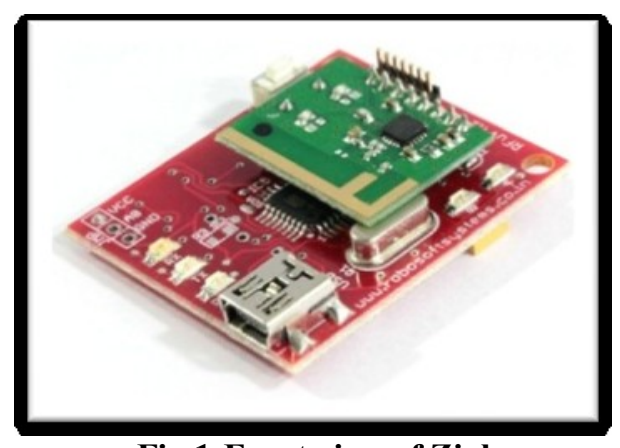

Fig 1:Front view of Zigbee

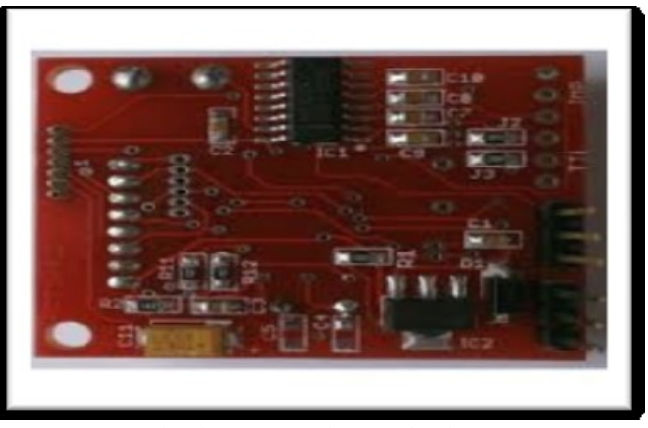

Fig 2:Back view of Zigbee

3) Comparative analysis of different technologies providing similar services [6]

\begin{tabular}{|l|l|l|l|}
\hline Category & Zigbee & Bluetooth & Wi-Fi \\
\hline Distance & $50-1600 \mathrm{~m}$ & $10 \mathrm{~m}$ & $50 \mathrm{~m}$ \\
\hline Power Supply & Years & Days & Hours \\
\hline Transmission Speed & $250 \mathrm{Kbps}$ & $1 \mathrm{Mbps}$ & $1.54 \mathrm{Mbps}$ \\
\hline Frequency Range & $\begin{array}{l}868 \mathrm{MHz}, \\
916 \mathrm{MHz}, \\
2.4 \mathrm{GHz}\end{array}$ & $2.4 \mathrm{GHz}$ & $2.4 \mathrm{GHz}$ \\
\hline Complicity & Simple & Complicated & Very complicated \\
\hline Extension & Automatic & None & $\begin{array}{l}\text { Depend on the existing } \\
\text { network }\end{array}$ \\
\hline Network Nodes & 65535 & 8 & 50 \\
\hline Cost of terminal unit & Low & Low & High \\
\hline Linking Time & $30 \mathrm{~ms}$ & Up to 10s & Up to 3s \\
\hline Security & $128 \mathrm{bit}, \mathrm{AES}$ & $64 \mathrm{bit}, 128 \mathrm{bit}$ & SSID \\
\hline Ease Of Use & Easy & Normal & Hard \\
\hline Prime Cost & Low & Low & Normal \\
\hline
\end{tabular}

4) Layout of zigbee modules (routers) in underground shaft mines

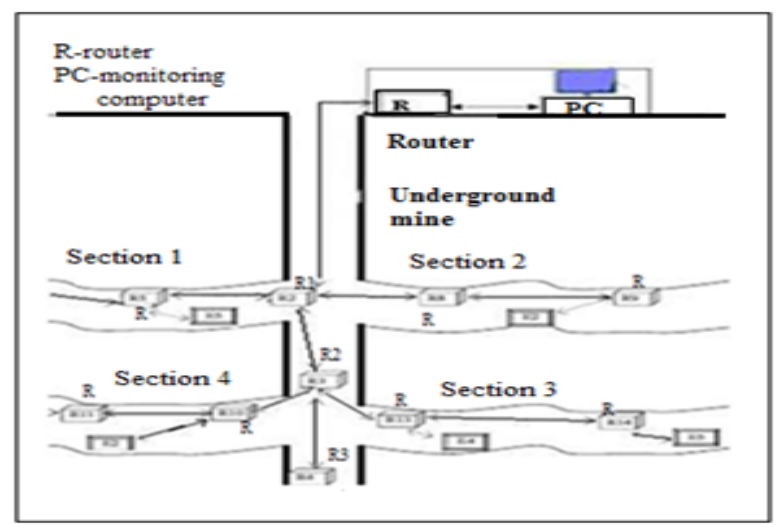

Fig.3: Layout of zigbee modules (routers) in underground shaft mines 


\section{1) Hardware Design at Local Site}

\section{Hardware Design}

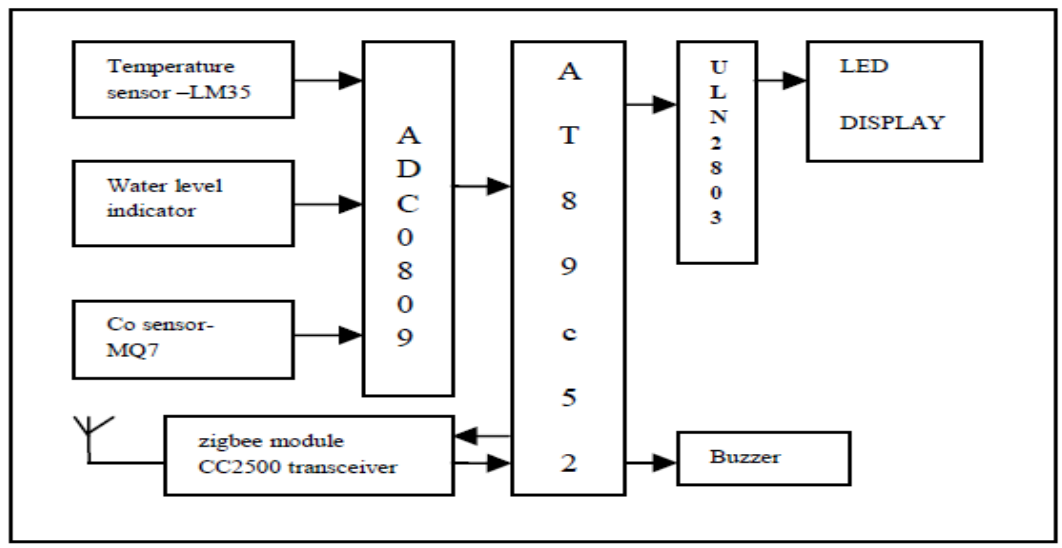

Fig.5: Block diagram of Hardware at local site

2) Implementation at remote monitoring site:

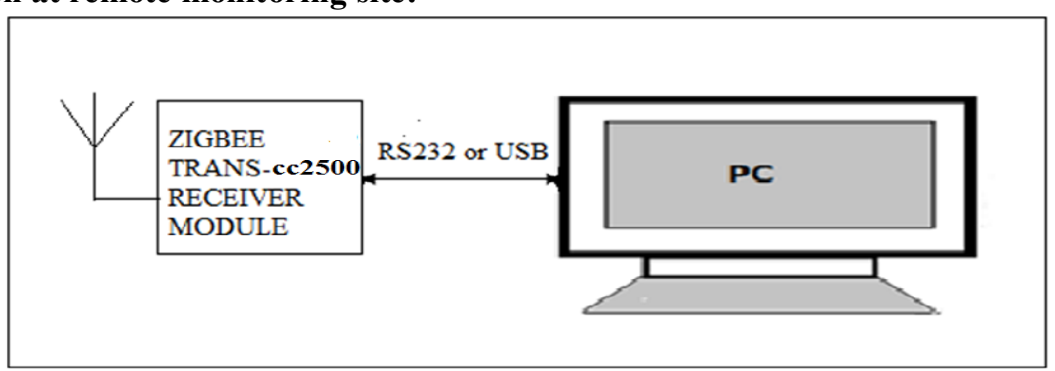

Fig.6: Block diagram of Hardware at remote monitoring site

\section{Software Used}

1) Proteus Software: For schematic circuit design, PCB design and simulation.

2) Keil $\mu$ vision Software: Keil provides a broad range of development tools like ansi c compiler, macro assemblers, debuggers and simulators, IDE, the real-time operating systems and evaluation boards for 8051, arm.

3) Embedded-C: $C$ is the most widely used programming language for embedded processors/controllers. Assembly is also used but mainly to implement those portions of the code where very high timing accuracy, code size efficiency, etc. are prime requirements.

4) Matlab: The language not only allows programmers to create simple GUI applications, but can also develop the complex applications.

\section{Description of The System}

As shown in block diagram 5, hardware at local site is composed of microcontroller AT89C52, Zigbee module CC2500 transceiver, sensors such as LM35 for temperature detection, water level indicator and MQ7 for carbon monoxide gas detection,ADC0809 IC,ULN2803 driver IC,LED segment display, buzzer for alert.

Various sensors like carbon monoxide MQ7,temperature sensor LM-35, water level detector are take analog measurement from local site of various parameter such as carbon monoxide, increasing temperature, different water level and through ADC give digital output to the microcontroller AT89C52.Zigbee transmits this digital sensor data to the remote monitoring site located at maximum distance from local site $(100 \mathrm{~m})$. Zigbee can be used inside mines at router as shown in figure 3. Remote monitoring site (pc) continuously monitoring sensor data in the designed format of GUI as shown in figure 3. Whenever the sensor data exceeds the specified threshold (preset) value of temperature, carbon monoxide and water level, the zigbee module at remote monitoring site is transmit alert signal to local site by blowing buzzer continuously and any one of the ' $\mathrm{G}, \mathrm{L}, \mathrm{C}$ ' alphabets will be displayed on LED segment according to the emerging situation ( $\mathrm{G}$ for gas, $\mathrm{L}$ for increasing water level, $\mathrm{C}$ for temperature).At remote monitoring site, GUI show different colours for different conditions. In case of temperature and carbon monoxide sense data, normal (safe condition) condition shown by green colour, for dangerous condition GUI show red colour. In case of water level detector, up to four levels the condition become normal and shown by green colour on GUI. After four layers, up to seven layer the condition become intermediate that is it give intimation that water level increased and goes toward danger situation. This 
is shown by yellow colour. The last condition is danger condition and shown by red colour. At this time, buzzer blowing continuously up to water level drop down in normal condition. In urgent situation, monitoring unit wants to call to any employee, then buzzer blow and the employee number displays on LED display. For example in bank account counter.

VII. Hardware Snap

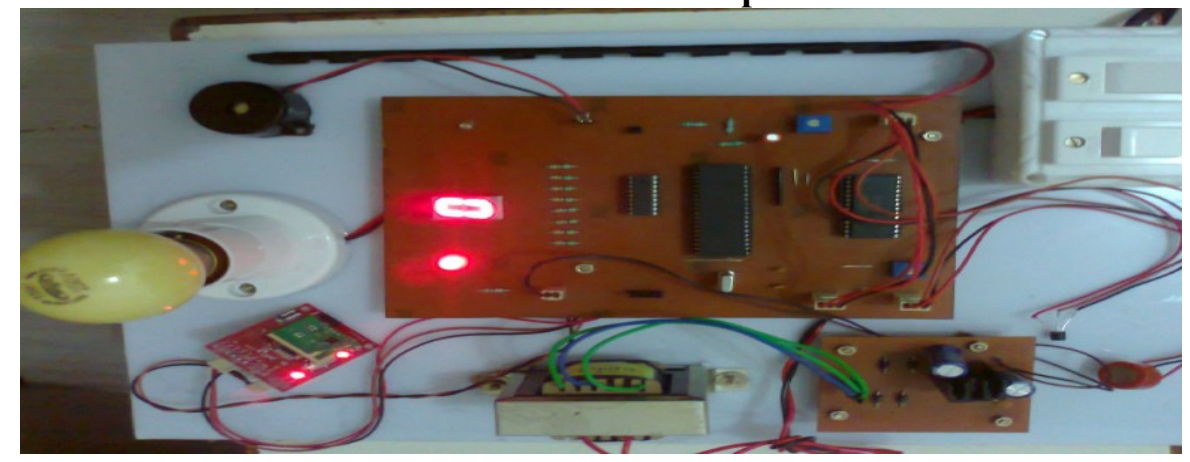

VIII. Software Implementation On Matlab Gui Screen Initial condition screen on matlab GUI screen

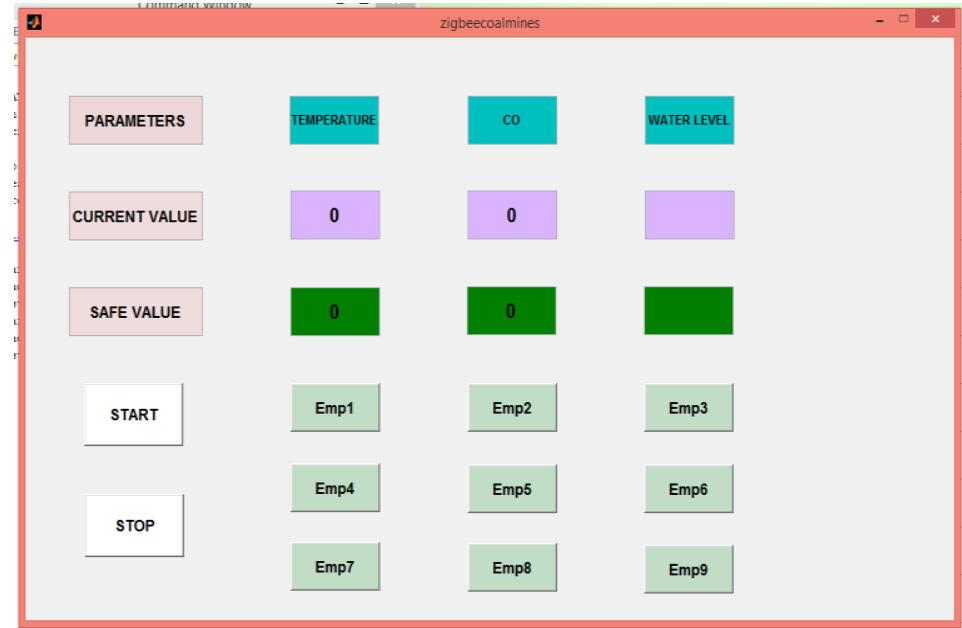

Danger condition for temperature

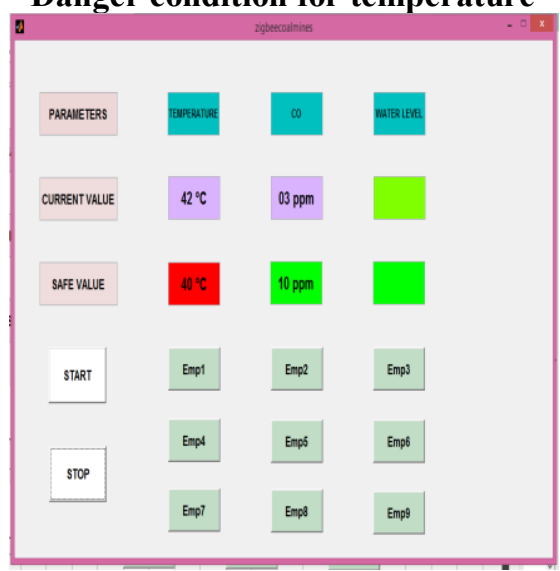

Danger condition for CO

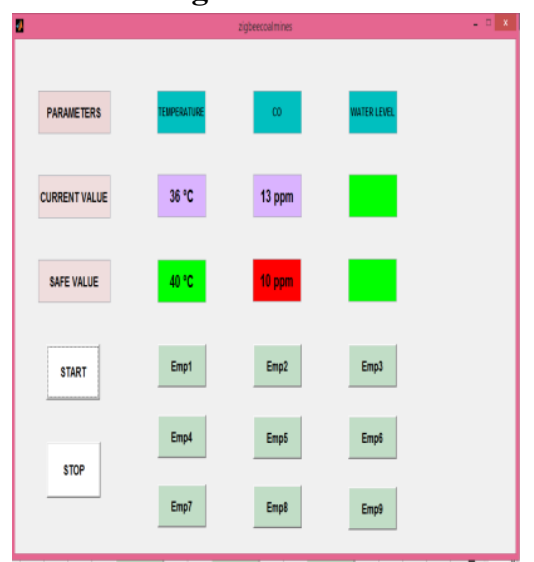



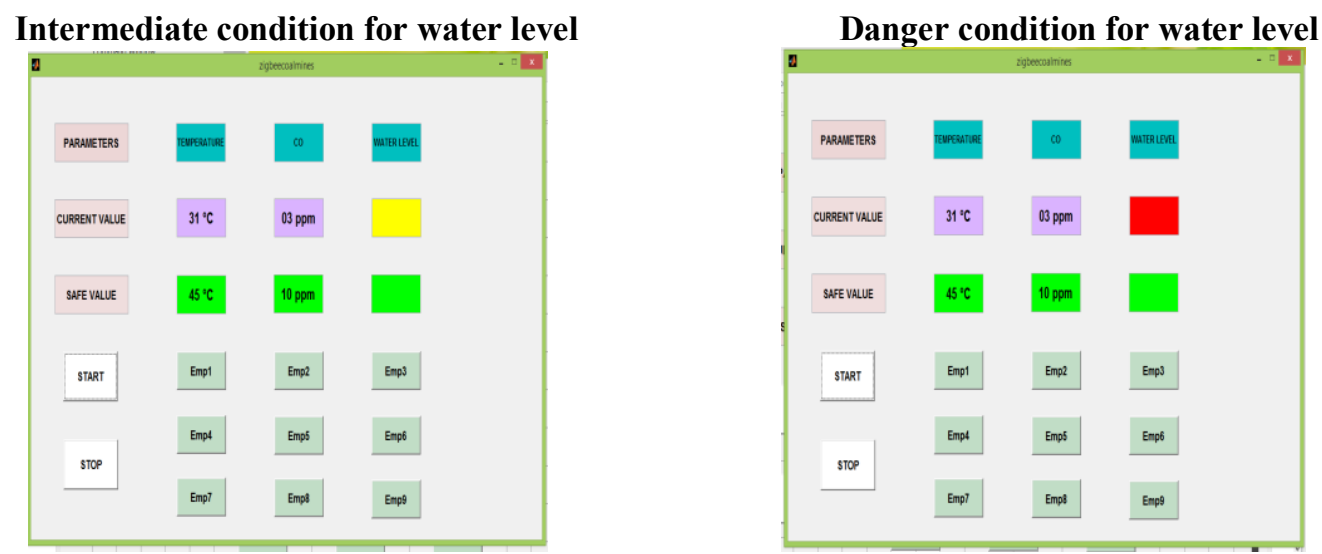

\section{Future Scope}

1. The system also can be easily extended with ZigBee wireless image transmission facility in future.

2. It will improve scalability of underground environment and extend accurate position of miners.

3. In future, with the help of Zigbee module and GUI (software part), we can avoid railways accidents, road accidents, submarine accidents etc.

\section{Conclusion}

The traditional mine security system can be effectively replaced by the surveillance and safety system proposed in the paper. This paper gives a system related to safety and security of underground mines. The system is reliable, faithful, uninterrupted, economical and user friendly. A larger area and more depth inside hazardous underground mines are now can be covered and accidents can be controlled effectively. The system combined the low power, low cost Zigbee based high frequency wireless data transmission technology. The sensor and zigbee module can be preferably installed in mines (routers).Proper monitoring and communication is possible between the employees and the monitoring site which can help to take appropriate actions more rapidly and smartly.

\section{Acknowledgements}

This work is supported by Silewara mines; Mines rescue services lab, W.C.L. Nagpur in practical study of underground mines environment. Also in future testing of all parameters of the presented system.

\section{References}

[1]. “The Research on ZigBee-Based Mine Safety Monitoring System”by Ge Bin, LI Huizong. 978-1-4244-8039-5/11[2011]

[2]. IEEE paper on "A Coal Mine Environmental Monitor System with Localization Function Based on ZigBee-Compliant Platform" by Dongxuan Yang, Yan Chen, Kedong Wang. 978-1-4244-6252-0/11/ C2011 IEEE

[3]. [3]"Design of the mine gas sensor based on zigbee" by Su baishun, Pang Zhengduo, MengGuoying [August 10].ISBN 978-952$5726-10-7$

[4]. IEEE paper on "Zigbee based intelligent helmet for coal miners" by CHENG Qiang, sun ji_ping, zhangzhe, zhang February [2009] CSIE2009.653

[5]. "Rescue and protection system for underground mine workers based on zigbee" by Tanmoymaity, parthasarathi das, mithumukherjee, vol.2 no.2 [June_December,2012] int.jr.of advanced computer eng.\& architecture

[6]. "Wireless Networking ThroughZigbee Technology" by P.Rohitha, P. Ranjeet Kumar Prof.N.Adinarayana, Prof.T.VenkatNarayanaRao ISSN: 2277 128X vol.2 [7 July 2012].

[7]. "Design And Development Of A SensorModel For Coalmines" bySrinivasa A.H1, G.Varaprasad

[8]. Application of gas monitoring sensors in underground coal mines and haradous area” by A. Kumar, T.M.G. Kingson, R.P. Verma, A. Kumar, R. Mandal, S. Dutta, S.K. Chaulya and G.M. Prasad CSIR-Central Institute of Mining and Fuel Research, Dhanbad, IndiaISSN 2249-6343 International Journal of Computer Technology and Electronics Engineering (IJCTEE) Volume 3, Issue 3, June 2013

[9]. "Challenges of gas monitoring and interpretation in underground coal mines following an emergency" by Peter Mason Coal Mines Technical Services, Mines Rescue Pty Ltd. University of Wollongong .Research Online Coal Operators' Conference Faculty of Engineering and Information Sciences $16-17$ February 2012 